\title{
Potential use of ultrasound for the detection of cell changes in cancer treatment
}
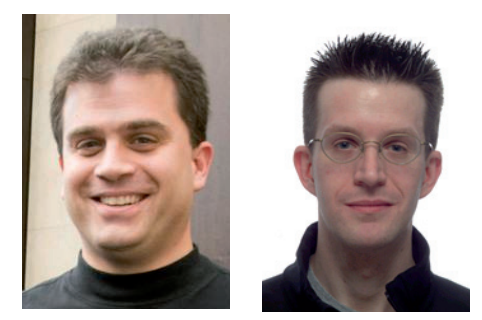

"Our work on ultrasound imaging of cell death indicates that ultrasound imaging and spectroscopy can potentially be used for the detection of both structural changes within tumor cells and morphologic changes in the gross tumor dimensions."

\section{Michael C Kolios ${ }^{\dagger}$ \& Gregory J Czarnota}

${ }^{\dagger}$ Author for correspondence: Department of Physics, Ryerson University, 350 Victoria Street, KHS-331C, Toronto, Ontario, M5B 2K3, Canada m Tel.: +1 4169795000 ext. 7065 m Fax: +1 4169795343 mmkolios@ryerson.ca

The era of personalized medicine, in which genomic and molecular data will be used to decide on the management of disease and the various treatment options, is thought to change the way oncology is practiced [1]. Specific assays could be used, based on the measurements of the levels of proteins or genes in individual patients, to devise tailored treatment strategies. Examples include the use of aromatase inhibitors and trastuzumab (Herceptin ${ }^{\circledR}$, Genentech Inc., CA, USA) for breast cancer and cetuximab for squamous cell carcinoma of the head and neck and colorectal cancer. However, these treatments will be at considerable societal and individual expense: brining a new drug to the market costs anywhere from US $\$ 500$ to US $\$ 2000$ million, depending on the therapy or the developing firm [2]. A method to assess treatment effectiveness during treatment, both in preclinical models and in a clinical setting, could have a significant impact. In this capacity, it is thought that "imaging will play an increasingly important role in the assessment of therapeutic response, especially in cancer" [1]. A recent review on imaging of therapeutic response highlights the various approaches that have been investigated [3].

To date, the most established methods for imaging cancer cell death in a potential clinical setting are the imaging of apoptosis with single photon emission computed tomography (SPECT) of radiolabeled annexin $\mathrm{V}$ [4-6] and the use of positron emission tomography ([PET]; using F-18 fluorodeoxyglucose [FDG]) to study the response of tumor metabolism after chemotherapy $[7,8]$. In the first method, the patient is injected with a radiopharmaceutical that binds to apoptotic cells. The second method relies on the injection of FDG and requires access to a
PET instrument. Cellular FDG uptake reflects the overall exchange rate of glucose, an indicator of cell viability. Despite the effectiveness of the method, it is expensive and requires multiple injections of a radioactive substance, reducing the technique appeal for longitudinal studies of treatment effectiveness. In general, a noninvasive technique that relies on endogenous contrast to evaluate treatment effectiveness would potentially have a much greater impact. Ultrasound is known to be a cost-effective imaging modality, which is rapid and portable with widespread access worldwide, thereby ensuring clinical impact for imaging procedures of demonstrated benefit. New generations of ultrasound devices exemplify the portability of these devices: in October 2009, General Electric released a pocket-sized ultrasound imaging device (the GE Vscan - for which the promotional material claims that this device can become 'the stethoscope of the 21st Century' [101]), and new ultrasound arrays are made that can attach to conventional 'smartphones' via a USB port and be used for ultrasound imaging [102], using the phone display to render the images.

The advantages of ultrasound led us to consider its use in a functional imaging capacity for cancer therapy, and resulted in our work on ultrasound imaging of apoptosis. Most people are familiar with ultrasound as an anatomical imaging modality, with functional information (mainly blood flow) that can be derived using Doppler techniques. However, ultrasound backscatter can provide information about cell structure that in turn is related to the functional state of cells: it has long been known that there is an increase in ultrasound backscatter in the necrotic centers of spheroids when imaged at $100 \mathrm{MHz}$ [9].

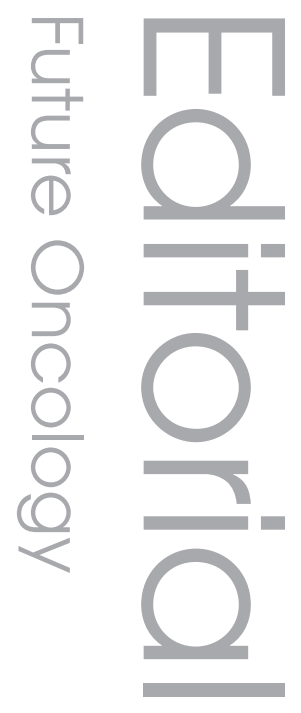

\section{future find $_{\text {Medicine }}$ fsg}


The authors of this study found that "The rim has a backscatter level similar to that of the cap, while the central region gives a surprising 20 -fold ( $25 \mathrm{~dB})$ increase in signal amplitude". This was a very large level of ultrasound contrast, which was not produced by the introduction of an exogenous contrast agent, as typically required by most other imaging modalities, but was produced by the very process of cell death itself. The regions of cell death in that study, determined histologically, correlated very well with the regions of increased backscatter. It should be noted that even at these high frequencies individual cells cannot be resolved, and the images produced have the typical speckle pattern seen in conventional ultrasound images. Therefore, it is the brightness of the speckle pattern that increased in regions where the cells responded to the treatment. That work indicated that high-frequency ultrasound, with wavelengths approaching the size of the cell, was potentially sensitive to the structural changes that cells undergo during cell death and, likely unbeknown to the authors at the time, apoptosis. Bérubé et al. studied the effects of nitromidazoles in multicellular spheroids [10]. Using an ultrasound imaging device (operating at $80 \mathrm{MHz}$ ), they also found that the ultrasound backscatter was greater in the necrotic core of spheroids. Significantly, they were able to observe the structural alteration of cells following the application of the cytotoxic drugs INO and $\mathrm{INO}_{2}$ in a timedependent fashion. They were able to measure the increase in ultrasound backscatter, as a function of time, in the interior of spheroids as the treatment time progressed.

In 1994 we started our work on the ultrasound imaging of apoptosis in particular. It was known that cells undergo dramatic structural changes during apoptosis. Therefore, we considered ultrasound imaging for preclinical applications initially and clinical applications, subsequently. To provide control over the level of apoptosis in the experiments, compact aggregates of cells were formed (what was often referred to in the publications as cell pellets - achieved though centrifugation). In a series of experiments, we found that there is a large change $(6-12 \mathrm{~dB})$ in ultrasound backscatter (frequencies of 20-60 MHz) for acute myeloid leukemia cells exposed to the chemotherapeutic cisplatin [11]. Similar experiments, as well as pilot experiments in vivo, demonstrated that this effect was robust: in a time-course experiment, we determined that apoptosis can be detected as early as $6 \mathrm{~h}$ after treatment $[12,13]$. The backscatter would increase and then subsequently decrease when the cells were in their advanced stages of programmed cell death and henceforth had lost most of their structure. Apoptotic cell death detection with ultrasound was also demonstrated during photodynamic therapy of the rat brain, with excellent correspondence of the region of increased backscatter to histology. To investigate the technique sensitivity, Tunis et al. used a centrifuged cell sample model and found that a mixture of $2.5 \%$ treated cells mixed with an untreated control population could be differentiated from an untreated control population, indicating that the technique is sensitive to low levels of apoptosis [13]. Finally, in recent work, it has been demonstrated that the technique can be applied in vivo, to differentiate between responding and nonresponding regions within a tumor $[14,15]$. These experiments were performed in xenograft tumor models: cells were injected intradermally into the hind leg of mice. These experiments demonstrated that even when other structures exist within the tumor (e.g., blood vessels or lymphatics), this method could still be used for treatment monitoring. This was an important step in establishing the methodology, since ultrasonic backscatter is dependent on the dominant scattering structures that give rise to the signal; it was not known if the presence of structures other than cells could reduce the technique sensitivity to the structural changes occurring during cell death.

Even though the ultrasound contrast between regions of cells responding to the treatment and regions that do not respond is large, variations in ultrasound backscatter alone can be very difficult to attribute to particular events or morphologies. For example, fat deposits in tissue can also appear to have an increased backscatter compared with the surrounding normal tissue parenchyma. The problem is compounded due to multiple instrument imaging parameters that can be modified during, and inbetween, imaging sessions, which could result in changes in image brightness. Moreover, it is difficult to compare images between different ultrasound machines and even for the same machine when different settings are used. This can be problematic when tumor response is to be determined by comparing a series of images that are acquired on different dates (and potentially instruments). To overcome many of these limitations, quantitative parameters based on the ultrasound backscatter that are independent of the instrument are calculated. These parameters are based on analyzing the backscatter radiofrequency (RF) echoes and include the integrated backscatter, RF envelope statistics, frequency dependence of the backscatter, ultrasound tissue attenuation and other signal 
classification techniques such as entropy metrics of RF ultrasonic backscatter [16,17]. The majority of work in quantitative ultrasound deals with tissue classification (e.g., the distinction between benign and malignant disease). We have applied these concepts for the differentiation between tissues that respond to cancer treatments and those that do not. For the monitoring of cell death in particular, we have investigated the use of the frequency dependence of the backscattered signals [18], the RF envelope statistics [13] and the integrated backscatter [19]. Analysis of the RF data provides additional information, which, together with the backscatter, can increase the technique sensitivity and specificity for the detection of the morphological changes associated with cell death.

It has long been known that the frequency dependence of the ultrasound backscatter can be related to the underlying tissue microstructure [20,21]. The spectral slope (the frequency content of the ultrasound backscatter normalized to a reference that contains the frequency content of the original interrogating ultrasound pulse) is a measure of the frequency dependence of the scattered ultrasound. For example, it is known that when the size of the scattering object is much smaller than the wavelength of the incident ultrasound the scattering intensity increases with the fourth power of frequency (the Rayleigh scattering regime). This frequency dependence is reduced when the size of the scattering object approaches the wavelength of the ultrasound. We have demonstrated that for purely apoptotic cell samples, the frequency dependence of the backscattered signal increases with cell death (an increase in the spectral slope of the normalized backscatter spectrum), which is attributed to the decrease in cell and nuclear size during apoptotic cell death $[18,22]$. Increases in the spectral slope were also measured in vivo for tumor responses in which the predominant mode of cell death appeared to be apoptosis $[14,22]$. The spectral slope remained relatively constant when there was a mixture of modes of cell death, and decreased in samples that predominantly underwent mitotic arrest/catastrophe [19,22]. This range of spectral slope changes is attributed to the structural changes that cells undergo during cell death: when mixed forms of cell death are present, in which the morphology associated with the cell death results in enlarged nuclei (e.g., mitotic arrest/catastrophe), then we have found that the spectra slope decreases [22]. Since ultrasound scattering and its frequency dependence is influenced primarily by nuclear structure in highly cellular tumors, the phenotypical changes in nuclear structure, as a function of time, will dictate the corresponding changes in spectral slope. Appropriate corrections for the intervening tissue attenuation would be required to properly interpret the spectral slope data, as it can also significantly affect these measurements $[23,24]$. Analysis of the frequency dependence of the ultrasound backscatter also has the potential to be used for tumor classification. In fact, a clear distinction between rat mammary fibroadenomas and 4T1 mouse carcinomas, which in ultrasound imaging alone cannot be differentiated, can be achieved by the use of quantitative ultrasound techniques [25].

Our work on ultrasound imaging of cell death indicates that ultrasound imaging and spectroscopy can potentially be used for the detection of both structural changes within tumor cells and morphologic changes in the gross tumor dimensions. Unlike other modalities being investigated for treatment monitoring, no injections of contrast agents are needed. This is the key advantage of this technique: the image contrast and changes in spectral power are caused by changes in the physical properties of the responding cells. But what are the limitations and challenges of using the technique in a clinical setting?

First, our theoretical and experimental data indicate that the technique sensitivity decreases with decreasing ultrasound frequency of interrogation. If higher frequencies are required $(>20 \mathrm{MHz})$ for adequate contrast between responding and nonresponding tissues, this limits the technique application to superficial tumors, as the imaging depth of ultrasound at $20 \mathrm{MHz}$ is a few centimeters. Whereas invasive probes at higher frequencies can be constructed to image tumors interstitially, in a manner somewhat similar to what is used for intravascular ultrasound [26], the technique invasiveness does not make it a suitable candidate for longitudinal imaging studies. Moreover, only a relatively small portion of the tumor would be imaged unless multiple insertions of the probe are made to cover larger areas of the tumor. In order to address this issue, recent work in our laboratories has indicated that differences between pellets of apoptotic and non-apoptotic cells can still be differentiated with frequencies as low as $5 \mathrm{MHz}$, albeit with a reduced contrast between the two cell types. Optimizing the ultrasound frequency band used in the experiments will depend on the tumor depth and what is known about its microstructure. This information can be used to make the appropriate ultrasound bandwidth selection that will involve a trade-off between penetration depth (lower frequency) and sensitivity to cell death 
(higher frequency). Our laboratories are currently focusing on determining the optimal such parameters for breast cancer. Second, whereas we have demonstrated that the technique can be used in human tumor xenografts transplanted subcutaneously in severely compromised immunodeficient (SCID) mice, it remains to be seen if the technique will still be effective in spontaneous tumors that may have different growth characteristics [27]. Moreover, it has been argued [28] that the tumor microstructure in subcutaneous xenografts can be quite different than that of human tumors, or tumor cell lines, and that orthotropic xenograft models may be required to better reproduce the histology and metastatic pattern of tumors. Since ultrasound scattering can be sensitive to higher order structures, the technique may be more sensitive to larger scattering structures in the tumors rather than the cells, as is the case when comparing mammary fibroadenomas and carcinomas [25]. This may potentially make the technique less sensitive to changes at the cellular level. Such experiments with orthotropic xenograft models are planned in the future.

Finally, another factor complicates the analysis of the backscattered data so as to accurately infer changes occurring in cell structure: irrespective of the acoustical properties of the individual tissue scatterers and how they may change during apoptosis/cell death, the spatial distribution of the scattering sources on its own is a source of large variations in ultrasound backscatter strength in the frequency band between 20 and $60 \mathrm{MHz}$ $[29,30]$. This suggests that the changes in ultrasound backscatter detected in our experiments can be at least partially attributed to the changes in the spatial organization of the cells as they undergo apoptosis. As the spatial distribution of scattering sources becomes more random (less correlated), backscattering increases. Since the histological samples of tumors indicates that the tumor cells before treatment have a more regular spatial distribution than after response to therapy, the increase in the cell spatial distribution randomness after treatment may contribute to the scattering increase observed. There is strong experimental evidence that this may be occurring during treatment response [22]. This phenomenon is somewhat similar to that of the dependence of ultrasound backscatter from blood as a function of hematocrit: initially, and as one would expect intuitively, ultrasound backscatter increases with concentration of red blood cells but, after a particular hematocrit, scattering monotonically decreases $[31,32]$. Since one of the goals of this work is to provide an estimate of the percentage of cells responding to the treatment, understanding this effect is important for the quantitative assessment of treatment response. Separating the individual contributions to the ultrasound backscatter of changes in scatterer acoustical properties and changes in scatterer spatial distribution has proven to be a difficult task and is an area of intense research activity in our laboratory. To this end, we have initiated experiments using acoustic microscopy and particle tracking microrheology to generate maps of acoustical properties of individual cells as a function of treatment response, so as to better understand the physical basis of the measurement and how to increase the method sensitivity and specificity using this new knowledge.

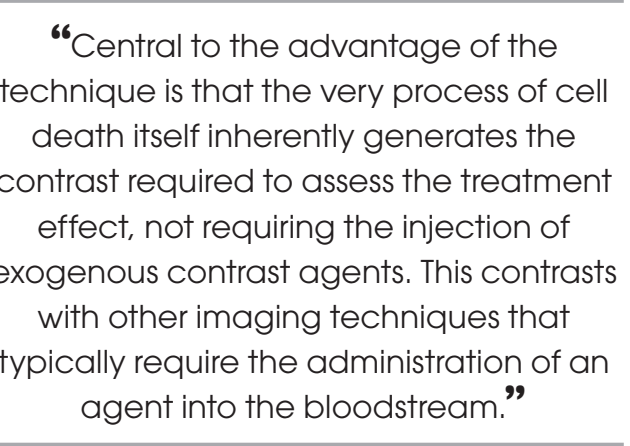

In summary, the applicability of using ultrasound imaging and spectroscopy to detect, noninvasively, cell death has been demonstrated in vitro and in vivo using a number of model tumor types and cell-death-inducing treatments. Central to the advantage of the technique is that the very process of cell death itself inherently generates the contrast required to assess the treatment effect, not requiring the injection of exogenous contrast agents. This contrasts with other imaging techniques that typically require the administration of an agent into the bloodstream that makes the approach less suitable for longitudinal studies of treatment response. As the process of cell death generates the contrast, to optimize the technique and understand its limitations, a comprehensive understanding of the physical basis of the measurement is required for specific tumor and cell phenotypes. The time kinetics of the contrast that is generated during cell death must be understood and quantified for the technique to be applied in a robust manner for treatment assessment, balancing trade-offs such as ultrasound frequency choice based on imaging penetration depth and detection sensitivity and specificity. Finally, another advantage of ultrasound imaging, not discussed in the context of this editorial, is that functional information of treatment response could also be gained 
by examination of the changes in the blood flow patterns though Doppler and power Doppler measurements. Our research is now focused on using both pieces of information for the complete characterization of the treatment response.

\section{Financial \& competing interests disclosure} Dr Michael C Kolios (MCK) holds a Tier 2 Canada Research Chair in Biomedical Applications of Ultrasound. Dr Gregory J Czarnota (GJC) holds a Cancer Care Ontario Research Chair in Experimental Therapeutics and Imaging. The research here was supported by grants from the Natural Sciences and Engineering Council of Canada and the Canadian Institutes of Health Research to both GJC and MCK, and infrastructure grants from the Canadian Foundation of Innovation, Ontario

\begin{abstract}
Ministry of Research and Innovation and Ryerson University. MCK and GJC are authors on an issued patent ('Detection of Apoptosis Using High-Frequency Ultrasound' US patent \#6511430) and a pending patent ('Methods of monitoring cellular death using low frequency ultrasound' US patent application \#11/455,005) held by the University Health Network (Toronto, Ontario, Canada) and licensed to VisualSonics Inc. (Toronto, Ontario, Canada). The authors have no other relevant affiliations or financial involvement with any organization or entity with a financial interest in or financial conflict with the subject matter or materials discussed in the manuscript apart from those disclosed.

No writing assistance was utilized in the production of this manuscript.
\end{abstract}

\section{Bibliography}

1. Thrall JH: Personalized medicine. Radiology 231(3), 613-616 (2004).

2. Adams CP, Brantner VV: Estimating the cost of new drug development: is it really 802 million dollars? Health Aff. (Millwood)25(2), 420-428 (2006).

3. Brindle K: New approaches for imaging tumor responses to treatment. Nat. Rev. Cancer 8(2), 94-107 (2008).

4. Belhocine T, Steinmetz N, Li C, Green A, Blankenberg FG: The imaging of apoptosis with the radiolabeled annexin v: optimal timing for clinical feasibility. Technol. Cancer Res. Treat. 3(1), 23-32 (2004).

5. Van De Wiele C, Lahorte C, Vermeersch H et al:: Quantitative tumor apoptosis imaging using technetium-99m-hynic annexin v single photon emission computed tomography. J. Clin. Oncol. 21(18), 3483-3487 (2003).

6. Belhocine TZ, Blankenberg FG: 99mtcannexin a 5 uptake and imaging to monitor chemosensitivity. Methods Mol. Med. 111, 363-380 (2005).

7. Kostakoglu L, Goldsmith SJ: PET in the assessment of therapy response in patients with carcinoma of the head and neck and of the esophagus. J. Nucl. Med. 45(1), 56-68 (2004).

8. Li S, Beheshti M: The radionuclide molecular imaging and therapy of neuroendocrine tumors. Curr. Cancer Drug Targets 5(2), 139-148 (2005).

9. Sherar MD, Noss MB, Foster FS: Ultrasound backscatter microscopy images the internal structure of living tumor spheroids. Nature 330(6147), 493-495 (1987).

10. Berube LR, Harasiewicz K, Foster FS, Dobrowsky E, Sherar MD, Rauth AM: Use of a high frequency ultrasound microscope to image the action of 2-nitroimidazoles in multicellular spheroids. Br. J. Cancer 65(5), 633-640 (1992).

11. Czarnota GJ, Kolios MC, Vaziri H et al.: Ultrasonic biomicroscopy of viable, dead and apoptotic cells. Ultrasound Med. Biol. 23(6), 961-965 (1997)

12. Czarnota GJ, Kolios MC, Abraham J, Portnoy M, Ottensmeyer FP, Hunt JW, Sherar MD: Ultrasound imaging of apoptosis: high-resolution non-invasive monitoring of programmed cell death in vitro, in situ and in vivo. Br. J. Cancer 81(3), 520-527 (1999).

13. Tunis AS, Czarnota GJ, Giles A, Sherar MD, Hunt JW, Kolios MC: Monitoring structural changes in cells with high frequency ultrasound signal statistics. Ultrasound Med. Biol. 31(8), 1041-1049 (2005).

14. Banihashemi B, Vlad R, Debeljevic B, Giles A, Kolios MC, Czarnota GJ: Ultrasound imaging of apoptosis in tumor response: Novel preclinical monitoring of photodynamic therapy effects. Cancer Res. 68(20), 8590-8596 (2008).

15. Vlad RM, Brand S, Giles A, Kolios MC, Czarnota GJ: Quantitative ultrasound characterization of responses to radiotherapy in cancer mouse models. Clin. Cancer Res. 15(6), 2067-2075 (2009).

16. Wallace KD, Marsh JN, Baldwin SL et al:: Sensitive ultrasonic delineation of steroid treatment in living dystrophic mice with energy-based and entropy-based radio frequency signal processing. IEEE Trans. Ultrason. Ferroelectr. Freq. Control 54(11), 2291-2299 (2007).

17. Hughes MS, Mccarthy JE, Marsh JN et al.: Properties of an entropy-based signal receiver with an application to ultrasonic molecular imaging. J. Acoust. Soc. Am. 121(6), 3542-3557 (2007).
18. Kolios MC, Czarnota GJ, Lee M, Hunt JW, Sherar MD: Ultrasonic spectral parameter characterization of apoptosis. Ultrasound Med. Biol. 28(5), 589-597 (2002).

19. Vlad RM, Alajez NM, Giles A, Kolios MC Czarnota GJ: Quantitative ultrasound characterization of cancer radiotherapy effects in vitro. Int. J. Radiat. Oncol. Biol. Phys. 72(4), 1236-1243 (2008).

20. Lizzi FL, Greenebaum M, Feleppa EJ, Elbaum M, Coleman DJ: Theoretical framework for spectrum analysis in ultrasonic tissue characterization. J. Acoust. Soc. Am. 73(4), 1366-1373 (1983).

21. Lizzi FL, Feleppa EJ, Astor M, Kalisz A: Statistics of ultrasonic spectral parameters for prostate and liver examinations. IEEE Trans. Ultrason. Ferroelectr. Freq. Control 44(4), 935-942 (1997).

22. Vlad RM: Quantitative ultrasound characterization of responses to radiotherapy in vitro and in vivo. Medical Biophysics, University of Toronto, Canada (2009) (Thesis).

23. Bigelow TA, O'Brien WD Jr: Impact of local attenuation approximations when estimating correlation length from backscattered ultrasound echoes. J. Acoust. Soc. Am. 120 (1), 546-553 (2006).

24. Bigelow TA, Oelze ML, O’Brien WD Jr: Estimation of total attenuation and scatterer size from backscattered ultrasound waveforms. J. Acoust. Soc. Am. 117(3 Pt 1), 1431-1439 (2005).

25. Oelze ML, O’Brien WD Jr, Blue JP, Zachary JF: Differentiation and characterization of rat mammary fibroadenomas and 4t 1 mouse carcinomas using quantitative ultrasound imaging. IEEE Trans. Med. Imaging 23(6), 764-771 (2004). 
26. Konig A, Margolis MP, Virmani R, Holmes D, Klauss V: Technology insight: in vivo coronary plaque classification by intravascular ultrasonography radiofrequency analysis. Nat. Clin. Pract. Cardiovasc. Med. 5(4), 219-229 (2008).

27. Kerbel RS: Human tumor xenografts as predictive preclinical models for anticancer drug activity in humans: better than commonly perceived-but they can be improved. Cancer Biol. Ther. 2 (4 Suppl. 1), S134-S139 (2003).

28. Garber K: Realistic rodents? Debate grows over new mouse models of cancer. J. Natl Cancer Inst. 98(17), 1176-1178 (2006).

29. Hunt JW, Worthington AE, Xuan A, Kolios MC, Czarnota GJ, Sherar MD: A model based upon pseudo regular spacing of cells combined with the randomisation of the nuclei can explain the significant changes in high-frequency ultrasound signals during apoptosis. Ultrasound Med. Biol. 28(2), 217-226 (2002).

30. Hunt JW, Worthington AE, Kerr AT: The subtleties of ultrasound images of an ensemble of cells: simulation from regular and more random distributions of scatterers. Ultrasound Med. Biol. 21(3), 329-341 (1995).

31. Mo LY, Cobbold RS: A unified approach to modeling the backscattered Doppler ultrasound from blood. IEEE Trans. Biomed. Eng. 39(5), 450-461 (1992).

32. Mo LY, Kuo IY, Shung KK, Ceresne L, Cobbold RS: Ultrasound scattering from blood with hematocrits up to $100 \%$. IEEE Trans. Biomed. Eng. 41(1), 91-95 (1994).

\section{Websites}

101. General Electric Reports: Vscan pocket-sized, ultra-smart ultrasound unveiled www.gereports.com/vscan-pocket-sized-ultrasmart-ultrasound-unveiled/

(Accessed 1 November, 2009)

102. Washington University (MO, USA): Ultrasound imaging now possible with a smartphone http://news-info.wustl.edu/tips/page/ normal/13928.html

(Accessed 1 November, 2009)

\section{Affiliations}

- Dr Michael C Kolios

Department of Medical Biophysics, University of Toronto, Ontario, Canada and

Department of Physics, Ryerson University, 350 Victoria Street, KHS-331C, Toronto,

Ontario, M5B 2K3, Canada

Tel.: +1 4169795000 ext. 7065

Fax: +1 4169795343

mkolios@ryerson.ca

- Dr Gregory J Czarnota

Departments of Radiation Oncology and Medical Biophysics, University of Toronto, Ontario, Canada and

Department of Radiation Oncology and Imaging Research, Sunnybrook Health Sciences Center, 2075 Bayview Avenue, Toronto, Ontario, M4N 3M5, Canada

Tel.: +14164806128

Fax: +1 4164806002

gregory.czarnota@sunnybrook.ca 